\title{
Negative index effects from a homogeneous positive index prism
}

\author{
Sherman W. Marcus ${ }^{1,2,}$ and Ariel Epstein ${ }^{2}$ \\ ${ }^{1}$ Rafael Advanced Defense Systems, Ltd., Haifa, Israel \\ ${ }^{2}$ Faculty of Electrical Engineering, Technion, Israel Institute of Technology, Haifa, Israel
}

Received: 4 September 2017 / Accepted: 25 October 2017

\begin{abstract}
Cellular structured negative index metamaterials in the form of a right triangular prism have often been tested by observing the refraction of a beam across the prism hypotenuse which is serrated in order to conform to the cell walls. We show that not only can this negative index effect be obtained from a homogeneous dielectric prism having a positive index of refraction, but in addition, for sampling at the walls of the cellular structure, the phase in the material has the illusory appearance of moving in a negative direction. Although many previous reports relied on refraction direction and phase velocity of prism structures to verify negative index design, our investigation indicates that to unambiguously demonstrate material negativity additional empirical evidence is required.
\end{abstract}

\section{Introduction}

Wave propagation through a medium with a negative index of refraction and across its boundaries has been of major interest in recent years. The problem was first hypothesized by Veselago [1], but became particularly relevant with the ability to create such "metamaterials". For example, negative permittivity can be achieved by arrays of wires [2], and negative permeability can be achieved by arrays of split ring resonators [3]. These cellular structures have been combined to produce metamaterials with a negative refraction index [4-7].

The properties of such cellular materials have been experimentally tested by shaping them into a right triangular prism, and observing a wave that propagates normal to one leg of the prism (represented by the arrow labelled "Incident ray" in Fig. 1 with $\theta_{i n c}=\theta_{p}$ ), is incident on the prism hypotenuse (the surface $\mathrm{AC}$ in Fig. 1), and is negatively refracted into a positive index medium (the arrow labelled "Negative refraction" in Fig. 1) [4-12]. Observation of this negatively refracted wave was deemed sufficient proof that the index of the prism material was negative. Such claims were supported by numerical simulations which demonstrated negative phase velocity within the material [9-11].

Although the typical metamaterial model describes the wave phenomena in the artificial material via an equivalent homogenized medium, in practice the device is made of unit

\footnotetext{
* e-mail: shermanwmarcus@gmail.com;

shermanm@technion.ac.il
}

cells of finite size. In order to avoid cutting through walls in the middle of such unit cells, the hypotenuse in experimental tests is usually serrated so that the material boundary coincides with these walls (surface AC in Fig. 1). It is well known that a plane wave crossing such a periodic boundary can result in a spectrum of waves compatible with both positive and negative refraction [13,14]. In this paper, we show (Sect. 2) that a serrated hypotenuse can produce a negatively refracted wave in any desired direction using a positive index prism material. Instead of a spectrum of waves, this negatively refracted wave would often be the only wave propagating across the boundary, thereby providing the impression that the material itself has a negative index of refraction. It is further shown (Sect. 3) that the field sampled at the cell walls within the positive index prism (i.e. at the locations of the red dots along the incidence arrow in Fig. 1) will exhibit an illusory negative phase velocity consistent with the desired negative refraction.

Section 4 applies the developments in Sections 2 and 3 to previously published measurement configurations of negative index designs. These are all shown to be compatible with positive index materials. This implies that experimental observations of negative refraction even accompanied by numerical measurements of negative phase velocity - are not necessarily indicative of a negative index material. In particular, our work reveals that wave scattering observations for a serrated prism are not sufficient for verifying negativity, and other evidence should be provided to establish the latter unambiguously. It should be emphasized that in experiments cited herein [4-12], such evidence has indeed been provided. 


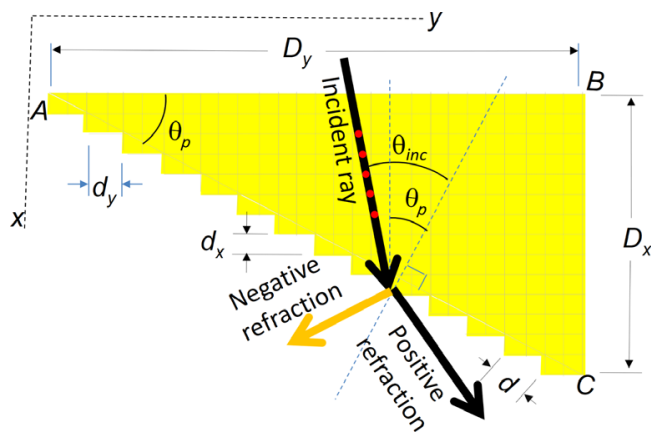

Fig. 1. Propagation through a cellular prism-shaped material with serrated hypotenuse. The red dots along the incident ray trajectory denote the intersection between the ray and the cell walls parallel to the $y$-axis where the phase is sampled to evaluate the phase velocity (see Sect. 3).

Although this study was prompted by the serrated geometry utilized in experiments to verify negative index designs, it suggests an extremely simple configuration for obtaining negative index effects using available materials. Negative effects from non-negative materials have previously been reported using photonic crystals [15-18]. These take advantage of the periodicity within the crystal material. In contrast, the negative index effects described in Sections 2 and 3 below apply to a positive-refractionindex prism-shaped material that is homogeneous, with the periodicity derived only from the surface of its hypotenuse. In other words, the same negative index phenomena can be observed with a simple geometry, comprised solely of bulk natural materials.

\section{Negative refraction}

Consider a two dimensional prism, characterized by a prism angle $\theta_{p}$, with a serrated hypotenuse with period $d$ (Fig. 1). When a plane wave is incident on that hypotenuse, plane waves will be transmitted into the air at angles $\theta_{m}$ given by the grating formula [19]:

$$
\sin \theta_{m}=n_{p} \sin \theta_{i n c}+\frac{m \lambda_{0}}{d}=n_{p} \sin \theta_{i n c}+\frac{m \gamma \lambda_{0} \sin \theta_{i n c}}{d_{x}},
$$

where $\lambda_{0}$ is the wavelength in air, $n_{p}$ is the index of the prism material, and $m$ is an integer denoting the order of the Floquet-Bloch mode. The factor $\gamma=\sin \theta_{p} / \sin \theta_{i n c}$ is indicative of the closeness of the incident wave direction to the direction normal to the leg $\mathrm{AB}$ of the prism, and $d_{x}=d \sin \theta_{p}$ is the lattice period in the $x$ direction (Fig. 1). To emulate an index of refraction $n_{c}$, we set

$$
n_{p}=\gamma \lambda_{0} / d_{x}+n_{c}
$$

which transforms (1) into

$$
\sin \theta_{m}=\left[(m+1) \gamma \lambda_{0} / d_{x}+n_{c}\right] \sin \theta_{i n c} .
$$

If the term in brackets is interpreted as an effective index of refraction, then (3) is in the form of Snell's law. For the $m=-1$ mode, this effective index of refraction will be $n_{c}$. If

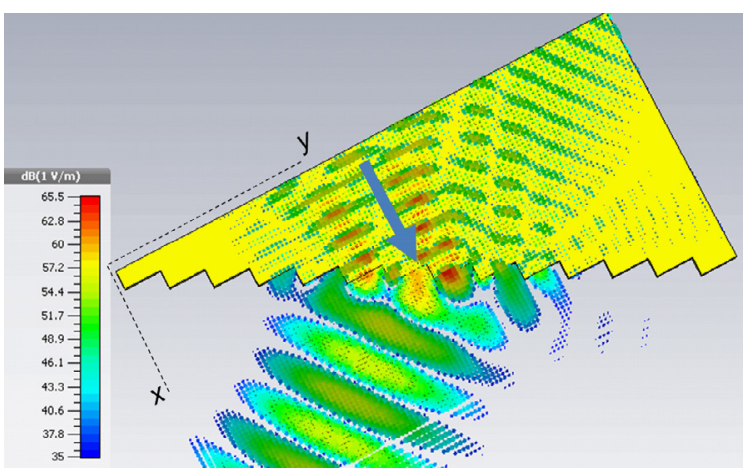

Fig. 2. CST E-field results for Gaussian beam incidence from the prism into the air. See Supplementary Material for an animated version of this figure.

$n_{c}$ is negative, then negative refraction will be emulated for this $m=-1$ mode even though the actual refraction index $n_{p}$ is positive. But this will be the case only if this $m=-1$ mode represents a propagation wave (and not an evanescent wave). Propagating waves will be produced only for values of $m$ for which $\left|\sin \theta_{m}\right|<1$. For $n_{c}<0$, the condition for only the $m=-1$ mode to propagate is

$$
\left|n_{c}\right|<1 / \sin \theta_{i n c}<n_{p}
$$

For example, assume it is desired to emulate a negative index $n_{c}=-1$ for a lattice characterized by $\lambda_{0} / d_{x}=4$, $D_{x} / D_{y}=1 / 2$ where $D_{x}, D_{y}$ are the lengths of the prism legs (Fig. 1), and for $\theta_{i n c}=\theta_{p}=\tan ^{-1}\left(D_{x} / D_{y}\right)=26.57$ so that $\gamma=1$. This can be accomplished from (2) by a prism with positive index $n_{p}=3$. From (3), waves will propagate when $|3+4 m|<2.24$ which can only be satisfied for $m=-1$, so that (4) is satisfied. Using the methods of [20], if $E_{i n c}$ is the amplitude of the incident wave and the prism relative permeability is unity, the "negatively refracted" wave amplitude will be $>0.8\left|E_{\text {ind }}\right|$ [21]. This less-than-optimal result is due to energy-backscatter from the periodic surface by a specularly reflected wave and by two grating lobes.

To verify these results for the above parameters, field computations using the finite-difference time-domain " $\mathrm{T}$ " solver of the CST Microwave Studio computer program [22] are shown in Figure 2 for a frequency of $10 \mathrm{GHz}$, with the leg length $D_{y}=7.5 \lambda_{0}$. The transverse electric $\left(E_{x}=\right.$ $\left.E_{y}=0\right)$ incident plane wave was simulated by a Gaussian beam the wave direction of which is indicated by the arrow in the figure. The only air-prism boundary accounted for in the computations was that across the prism hypotenuse.

This single wave in the air would appear as if it had been "negatively refracted" in accordance with (3) even though it had originated in a positive index material. The source of this "refraction" is, of course, the periodic geometry of the hypotenuse which, as indicated, is present in most experimental tests of negative material designs [4-12]. Although this periodic geometry derives from the assumed cellular form of the prism, this cellular form plays no other role in the computations, so the material can be assumed homogeneous. 
Table 1. Measurement configurations for negative material design verification, and computed positive materials for producing the same negative refraction characteristics.

\begin{tabular}{lllllll}
\hline Ref. & $\lambda_{0}$ & $d_{x}$ & $\theta_{p}\left(^{\circ}\right)$ & $n_{c}$ & $n_{p}=n_{p}^{\prime}$ & $m$ \\
\hline$[5]$ & $23.8 \mathrm{~mm}^{\mathrm{a}}$ & $3.3 \mathrm{~mm}^{\mathrm{b}}$ & 32.2 & -1.05 & 6.16 & -1 \\
{$[6]$} & $21 \mathrm{~mm}^{\mathrm{a}}$ & $3.3 \mathrm{~mm}^{\mathrm{b}}$ & 12 & -0.8 & 5.56 & -1 \\
{$[7]$} & $28.6 \mathrm{~mm}$ & $6 \mathrm{~mm}$ & 18.4 & -0.35 & 4.41 & $-1^{\mathrm{c}}$ \\
{$[7]$} & $28.6 \mathrm{~mm}$ & $6 \mathrm{~mm}$ & 26.6 & -0.35 & 4.41 & $-1^{\mathrm{c}}$ \\
{$[9]$} & $5.6 \mathrm{~mm}$ & $1.5 \mathrm{~mm}$ & 26.6 & -1 & 2.73 & -1 \\
{$[9]$} & $5.22 \mathrm{~mm}$ & $1.5 \mathrm{~mm}$ & 26.6 & -0.2 & 3.28 & -1 \\
{$[11]$} & $1748 \mathrm{~nm}^{\mathrm{a}}$ & $75.2 \mathrm{~nm}$ & 5 & -1 & 22.2 & -1 \\
{$[12]$} & $28.6 \mathrm{~mm}$ & $5 \mathrm{~mm}^{\mathrm{b}}$ & 20 & -0.55 & 5.16 & -1 \\
\hline
\end{tabular}

${ }^{\mathrm{a}}$ A spectrum of $\lambda_{0}$ values was employed; one was chosen as representative.

b Not specified whether hypotenuse was serrated.

${ }^{\mathrm{c}}$ Two separate waves were measured.

It is worth noting that the observed effect is not limited to cellular structures that obey homogenization $\left(\lambda_{0} / d_{x} \gg 1\right)$, which are the focus of this paper. Equations (2)-(4) are equally applicable to smaller values of $\lambda_{0} / d_{x}$ with appropriate values of $n_{c}$ and $\theta_{i n c}$.

\section{Negative phase velocity}

Even though the propagation described by (3) and illustrated in Figure 2 can produce a single wave with negative refraction characteristics, the phase velocity of the incident wave within the prism will be positive since $n_{p}>1$. Nevertheless, it will now be shown that simulation of this incident wave can produce the appearance of negative phase velocity.

The prism in Figure 1 is divided into imaginary cells which are filled with a material with a positive index $n_{p}^{\prime}$, the value of which, for the time being, will be assumed unknown. We are interested in the phase of the wave at the points indicated by red dots along the incident ray of Figure 1. Assuming an $\exp (-i \omega t)$ time dependence where $\omega$ is the angular frequency, the change of phase of the incident wave over a single cell row of thickness $d_{x}$ (i.e. from one red dot to the next in the direction of the incident ray in Fig. 1) will be

$$
\Delta \varphi_{p}=k_{0} n_{p}^{\prime} d_{x} / \beta
$$

where $k_{0}=2 \pi / \lambda_{0}$ is the wave number in free space, and $\beta=\cos \left(\theta_{i n c}-\theta_{p}\right)$. Similarly, for a prism with negative index $n_{c}$, this change of phase would be negative:

$$
\Delta \varphi_{c}=k_{0} n_{c} d_{x} / \beta
$$

But, because of the equivalence of phase angles that differ by integer multiples of $2 \pi$, this negative phase difference is equivalent to the positive phase difference

$$
\Delta \varphi_{e}=\Delta \varphi_{c}+2 \pi
$$

But this positive phase difference can be achieved in a positive index medium in accordance with (5). The refractive index $n_{p}^{\prime}$ of that medium can therefore be obtained by equating (5) with (7):

$$
n_{p}^{\prime}=\beta \lambda_{0} / d_{x}+n_{c}
$$

Therefore, the phase of a wave in a material with negative index $n_{c}$ can be emulated on the discrete lattice walls by a homogeneous material with positive index $n_{p}^{\prime}$ given by (8). To clarify this, consider as before $n_{c}=-1, \lambda_{0} / d_{x}=4$, $\theta_{i n c}=\theta_{p}$, so that $n_{p}^{\prime}=3$. The incident waves within materials characterized by $n_{p}^{\prime}$ and $n_{c}$ would be given by $\cos \left(k_{o} n_{p}^{\prime} x-\omega t\right)$ and $\cos \left(k_{0} n_{c} x-\omega t\right)$, and are illustrated in Fig. 3 for several values of $\omega t$. Although these waves move in opposite directions since the signs of $n_{p}^{\prime}$ and $n_{c}$ are opposite, the phase of both waves coincides along the lattice walls (vertical lines in Fig. 3) at every instant $\omega t$. Therefore, if the phase of the wave in the positive medium (solid curves) is sampled only on the lattice walls, it would appear to be moving in the negative direction, particularly since there are more sampled points per wavelength for the negative wave than for the positive wave. (See Supplementary Material for a dynamic demonstration of this phenomenon).

This observation is important since investigators have typically employed numerical simulations within a proposed negative prism to supplement wave measurements outside it [9-11]. However, as shown, if the in-prism field is naively sampled at lattice intervals, the phase would appear negative even in a positive medium. Thus, to properly verify negative phase velocity, one has to be aware of this "illusion", and tune the sampling resolution to suitable values.

\section{Prism experiments}

For a given $\theta_{\text {inc }}$, the $n_{p}^{\prime}$ required to emulate the phase by $n_{c}$ material in (8) is generally different from the $n_{p}$ required to emulate refraction by $n_{c}$ material in (2). An exception to this is when $\theta_{i n c}=\theta_{p}$, which is precisely the incidence angle used in experimental material tests, since it corresponds to a wave direction that is normal to leg $\mathrm{AB}$ (Fig. 1). This implies $\gamma=\beta$ and $n_{p}=n_{p}^{\prime}$, so that, in principle, these tests can appear to produce both negative refraction and negative phase velocity consistent with the same negative-refraction-index even if the material were positive.

Table 1 provides examples of the application of these principles to published measurements of negative index designs using prism-shaped materials. Each entry in the 


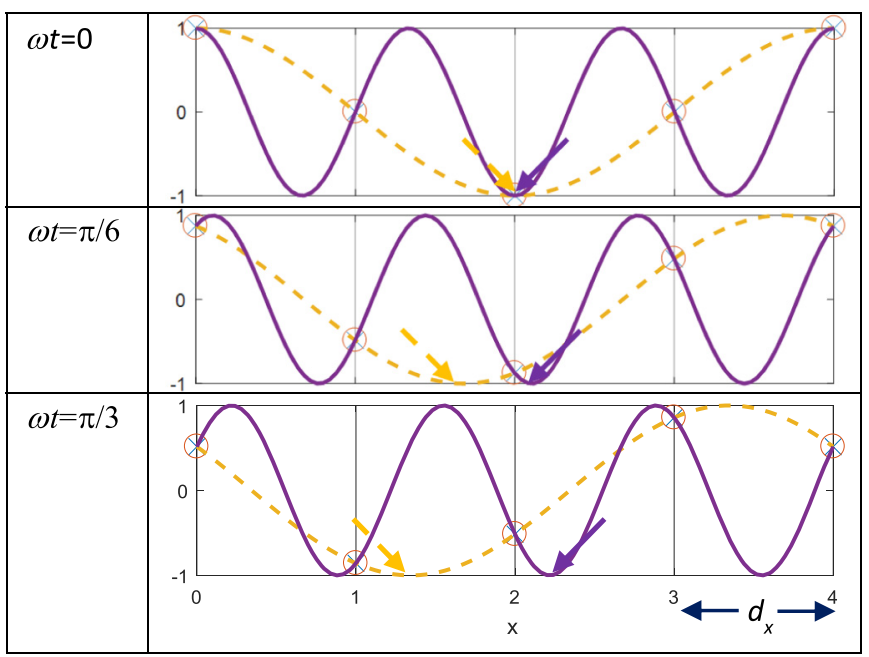

Fig. 3. The equivalence along cell boundaries of the phase in a negative medium (dashed curve moving leftward, $n_{c}=-1$ ) and the phase in a positive medium (solid curve moving rightward, $n_{p}^{\prime}=3$ ). An arrow indicates an extremum for each wave at each time. See Supplementary Material for an animated version of this figure.

table contains the relevant parameters employed in the measurements documented in the reference listed in the first column, and with the observed refraction index $n_{c}$. These were used to compute from (2) the positive index $n_{p}$ which would produce the same negatively refracted wave across the serrated surface, and the indices $m$ which satisfy $\left|\sin \theta_{m}\right|<1$ using (3) thereby assuring that the corresponding wave is propagating and not evanescent.

The most striking outcome of Table 1 is that not only are all measurement configurations compatible with an $m=-1$ "negatively-refracted-wave" for propagation from a positive material across a serrated boundary, but that wave is the only wave predicted to propagate. For all measurements, a single negatively refracted wave was observed. However, in [7] an additional positively refracted wave was also observed contrary to predictions.

It should be emphasized that the ability to reproduce the measurements using a positive index material does not imply that the materials in the cited references are not negative. On the contrary, these references do provide additional evidence to support negative material claims. However, it does demonstrate that refraction direction measurements are generally not sufficient to prove a material is negative.

\section{Conclusions}

We have shown that negative refraction effects can be obtained from a relatively simple configuration of a right triangular prism with a serrated hypotenuse. The prism material is composed of a homogeneous isotropic dielectric, the positive index of which depends on the negative refraction index $n_{c}$ that is to be emulated, and on the ratio of the wavelength to the cell size. This negative refraction is complemented by an illusory negative phase velocity consistent with $n_{c}$ - along virtual transverse cell walls within the material.

Although many previous reports relied on refraction direction measurements and on field simulations to verify negative index design, our investigation shows that additional empirical evidence is required to unambiguously prove material negativity. It is therefore essential that negative index effects be distinguished from negative index materials.

\section{Supplementary Material}

Supplementary files supplied by authors. The Supplementary Material is available at https://epjam. edp-open.org/10.1051/epjam/2017011.

\section{References}

1. V.G. Veselago, Sov. Phys. Usp. 10, 509 (1968)

2. J.B. Pendry, A.J. Holden, D.J. Robbins, W.J. Stewart, J. Phys. Condens. Matter 10, 4785 (1998)

3. J.B. Pendry, A.J. Holden, D.J. Robbins, W.J. Stewart, IEEE Trans. Microw. Theory Tech. 47, 2075 (1999)

4. R.A. Shelby, D.R. Smith, S. Schultz, Science 292, 77 (2001)

5. C.G. Parazzoli, R.B. Greegor, K. Li, B.E.C. Koltenbah, M. Tanielian, Phys. Rev. Lett. 90, 107401 (2003)

6. R.B. Greegor, C.G. Parazzoli, K. Li, B.E.C. Koltenbah, M. Tanielian, Opt. Express 11, 688 (2003)

7. A.A. Houck, J.B. Brock, I.L. Chuang, Phys. Rev. Lett. 90 , $137401(2003)$

8. M. Navarro-Cía, M. Beruete, F. Falcone, M. Sorolla, Prog. Electromag. Res. Vol. PIER 103, 139 (2010)

9. M. Navarro-Cía, M. Beruete, M. Sorolla, I. Campillo, Opt. Express 16, 560 (2008)

10. J. Valentine, S. Zhang, T. Zentgraf, X. Zhang, Proc. IEEE 99, 1682 (2011).

11. J. Valentine, S. Zhang, T. Zentgraf, E. Ulin-Avila, D.A. Genov, G. Bartal, X. Zhang, Nature 455, 376 (2008)

12. M.C. Velazquez-Ahumada, M.J. Freire, J.M. Algarin, R. Marques, Am. J. Phys. 79, 4 (2011)

13. D.R. Smith, P.M. Rye, J.J. Mock, D.C. Vier, A.F. Starr, Phys. Rev. Lett. 93, 130401 (2004)

14. S. Larouche, D.R. Smith, Opt. Lett. 37, 2391 (2012)

15. C. Luo, S.G. Johnson, J.D. Joannopoulos, J.B. Pendry, Phys. Rev. B 65, 20 (2002)

16. C. Luo, S.G. Johnson, J.D. Joannopoulos, J.B. Pendry, Opt. Express 11, 7 (2003)

17. A. Martínez, J. Martí, Phys. Rev. B 71, 235115 (2005)

18. A. Martínez, J. Martí, Opt. Express 14, 6755 (2006)

19. D. Maystre, Scholarpedia 7, 11403 (2012)

20. R.A. Depine, A. Lakhtakia, Phys. Rev. E 69, 057602 (2004)

21. The computer program on which the results of this paper are based is provided at http://arielepstein.webs.com/Software/ PASS.zip. It relies on Floquet-Bloch mode expansions [20], and computes the direction and amplitude of each propagating mode and the amplitude of each evanescent mode.

22. www.cst.com

Cite this article as: Sherman W. Marcus, Ariel Epstein, Negative index effects from a homogeneous positive index prism, EPJ Appl. Metamat. 2017, 4, 9 